\title{
Exoearth study with TMT
}

\section{Richard G. Dekany}

Richard G. Dekany, "Exoearth study with TMT," Proc. SPIE 5382, Second Backaskog Workshop on Extremely Large Telescopes, (7 July 2004); doi: 10.1117/12.566101

SPIE Event: Second Backaskog Workshop on Extremely Large Telescopes, 2003, SPIE. Backaskog, Sweden 


\title{
Exoearth study with TMT
}

\author{
Richard Dekany \\ Caltech Optical Observatories, M/S 105-24 \\ California Institute of Technology, Pasadena, CA 91125
}

\begin{abstract}
Ground-based optical and infrared telescopes with diameters of 30-meters or greater have theoretical potential to study objects at the contrast levels predicted for reflecting terrestrial planets in orbits within the habitable zone of nearby stars. Despite the corrupting effect of the Earth's turbulent atmosphere, the theoretical limits can be approached through the use of an adaptive optics ( $\mathrm{AO}$ ) system optimized for high contrast operating at near-infrared wavelength. With proper flow-down of functional requirements and contrast-optimized choice of site, the highly segmented Thirty Meter Telescope (TMT) could study scores of nearby star systems, to apparent magnitude 5 , for resident terrestrial planets at spectral resolution $R=5$ in either visible or near-infrared band, and a few systems, to magnitude 3 , at $R=20$ in the infrared. Even at low spectral resolution, a wealth of information could be obtained by direct imaging of exoearths, including determination of the presence of an atmosphere, clouds, equilibrium temperature, tidal locking, and the presence of non-Earth-like atmospheric chemistry such as steam lines. Our own atmosphere, however, limits the study of exoearth biological markers, unless these planets have environmental conditions and chemical composition significantiy different from our own.
\end{abstract}

\section{INTRODUCTION}

The dual advantages of increasing light gathering power and background-reducing image resolution make large telescopes ideal machines to study nearby extrasolar planetary systems. In the absence of any other effects, the required integration time from such studies would scale as telescope diameter, D, raised to the fourth power. Ground-based telescopes, however, must contend with a host of interfering effects. Foremost among these are diffraction, sky background, molecular and aerosol scatter, and residual phase and amplitude wavefront errors that scatter light from the bright parent star and can potentially obscure the desired planet signal.

For reference, a solar analogue at a distance of $10 \mathrm{pc}$ would have an apparent magnitude that is a function of observing band: $\mathrm{m}_{\mathrm{R}}=4.7, \mathrm{~m}_{\mathrm{H}}=3.2$, where $\mathrm{R}$-band is defined here at having mean wavelength $\lambda_{\mathrm{R}}=0.7 \mu \mathrm{m}$ and $31 \%$ bandwidth and $\mathrm{H}$-band as having $\lambda_{\mathrm{H}}=1.65 \mu \mathrm{m}$ and $18 \%$ bandwidth. This would produce stellar fluxes of $27 \times 10^{7}$ photons $/ \mathrm{m}^{2} / \mathrm{s}$ and $13 \times 10^{7}$ photons $/ \mathrm{m}^{2} / \mathrm{s}$ at the top of the Earth's atmosphere, respectively. Detected flux levels must include transmission losses. An Earth analogue in such a system would have a reflection contrast of $1.7 \times 10^{-9}$, assuming a geometric albedo of 0.33 and a 90 degree phase angle. The corresponding exoearth flux would therefore be 0.46 and 0.22 photons $/ \mathrm{m}^{2} / \mathrm{s}$ for R and H-band respectively. TMT, with a collecting aperture of approximately 700 square meters, would collect 322 and 154 photons/s in $\mathrm{R}$ and $\mathrm{H}$, before losses, demonstrating the theoretical potential for exoplanet study well beyond $10 \mathrm{pc}$.

The habitable zone ${ }^{1}$ for such a Sun-Earth analogue system would extend from approximately 0.05 " to 0.2 " in angular radius from the star. For stars of different temperature and distance, this range is expanded to approximately $0.003^{\prime \prime}$ to $0.3^{\prime \prime}$ for nearly all stars within $15 \mathrm{pc}$.

\section{LIMITATIONS TO GROUND AO CORONAGRAPHY}

\subsection{Diffraction}

Suppression of light diffracted by the collecting aperture and any intervening obscurations (including intersegment gaps in the primary mirror) is essential. Fortunately, many approaches have been proposed that exceed the suppression capability of the classical coronagraph by Lyot ${ }^{2}$, including variable transmission occulting masks ${ }^{3,4}$, Sonine-apodized 
pupils $^{5,6}$, shaped-pupils ${ }^{7}$, nulling beamcombiners ${ }^{8,9}$, and pupil mapping ${ }^{10,11}$. These techniques, or perhaps more than one in combination, will allow sufficient suppression with an idealized pupil, suffering only potential planet signal throughput losses.

The issues for a highly segmented telescope primary are not significantly worse in the case of exoearth study. This is because the diffraction pattern imposed upon the point spread function (PSF) by the many intersegment gaps and edges, a characteristic hexagonal-symmetric 'forest' of spikes, can be made to have a minimum angular extent outside the habitable zone. For H-band, and segments of (flat-to-flat) diameter of $d_{s}=0.9$ meters, the first diffraction spot from segmentation occurs at an angular radius $\lambda / \mathrm{d}_{\mathrm{s}}=0.378$ ". Errors in segment alignment across the primary mirror could, however, degrade performance, but a well-designed AO system can compensate from all residual wavefront errors having spatial frequencies of interest. In other words, the uncorrectable phase errors due to segmentation corrupt the PSF far outside the habitable zone. Because transmission losses could be severe for certain primary mirror segmentation approaches, the impact on high-contrast coronagraphy should be considered an integral part of the TMT design.

\subsection{Sky background and molecular and aerosol scatter}

The thermal emission of photons from the Earth's atmosphere adds a noise source to the study of exoplanets. For a good astronomical site, the sky brightness is typically 21.5 and 16 magnitudes $\operatorname{arcsec}^{-2}$ at $\mathrm{R}$ and $\mathrm{H}$-band respectively. For an exoearth of brightness $m_{R}=29.1$ and $m_{H}=27.6$, the sky flux in a diffraction-limited resolution element will be some 2.7 mags fainter than the planet signal in $\mathrm{R}$, but 3.1 mags brighter than the planet in $\mathrm{H}$. Moreover the broad scattered light halo due to molecular and aerosol scattering can also produce 'background' signals that potentially overwhelm the planet signal. The strength of the scatter halo is a strong function of local atmospheric conditions, and careful aerosol abundance criteria must be employed. In some cases for R-band, the molecular scatter halo has been shown to have a surface brightness of $10^{-6}$ that of the parent $\operatorname{star}^{12}$. However, disentanglement of this effect from residual telescope figure errors on intermediate spatial scales for large telescopes has been difficult ${ }^{13}$.

In theory, because of their spatially smooth profile, sky and molecular scatter background signals could be removed entirely. In reality, photon noise from each source, and limitations on one's ability to flat-field one's science detector, will limit the efficacy of subtraction. In the worse case, assuming an ability to flat-field to $0.03 \%{ }^{14}$, both the $\mathrm{H}$-band sky background and R-band scattered light halo may set a limit of contrast around $3 \times 10^{-10}$. Although comparable to the exoearth contrast, new techniques for subtraction of smooth haloes will be required. While these are not fundamental limitations, they will require advancements in the state-of-the-art to overcome.

\subsection{Residual wavefront errors}

Table 1 lists key physical processes that result in residual wavefront errors, potential technological mitigations, the technological maturity of the proposed mitigation, and information pertaining to the nature, size, and decorrelation time of PSF halo speckles induced by each process. For some error processes, more than one mitigation technique may be applied while it is likely that a well-optimized ground-based AO system will require a mitigation strategy for each.

\subsubsection{Second-order wavefront expansion}

Sivaramakrishnan ${ }^{15}$ has expanded the scalar wavefront for small residual phase errors to second-order, $\Psi(\mathrm{x}, \mathrm{y})=\mathrm{A} \mathrm{e}^{\mathrm{i} \phi(\mathrm{x}, \mathrm{y})}$ $\sim \mathrm{A}\left(1+\mathrm{i} \phi-\phi^{2} / 2\right)$, to write the following description of the point spread function,

$$
\begin{gathered}
a a^{*} \\
+(a \otimes \Phi)\left(a^{*} \otimes \Phi^{*}\right) \\
+\left\{2 \operatorname{lm}\left[a\left(a^{*} \otimes \Phi^{*}\right)\right]-\frac{1}{2}\left[a\left(a^{*} \otimes \Phi^{*} \otimes \Phi^{*}\right)+a^{*}(a \otimes \Phi \otimes \Phi)\right]\right\}
\end{gathered}
$$

where the circle-cross symbol denotes convolution and Im is the imaginary part. 


\begin{tabular}{|c|c|c|c|}
\hline Effect & Potential mitigation & $\begin{array}{c}\text { Mitigation } \\
\text { maturity }\end{array}$ & $\begin{array}{c}\text { Halo width / } \\
\text { Decorrelation time }\end{array}$ \\
\hline Boiling wind lag & $\begin{array}{l}\text { High closed-loop correction } \\
\text { bandwidth }\end{array}$ & Insufficient & $\begin{array}{c}\text { Seeing disk }\left(\lambda / \mathrm{r}_{0}\right) \\
\text { Atm. boiling time (typ. } 200 \mathrm{msec})\end{array}$ \\
\hline Chromatism & $\begin{array}{l}\text { Site selection } \\
\text { Atmospheric monitoring } \\
\text { Common-band wavefront } \\
\text { sensing } \\
\end{array}$ & $\begin{array}{l}\text { High } \\
\text { Moderate } \\
\text { Moderate }\end{array}$ & $\begin{array}{c}\text { Seeing disk }\left(\lambda / \mathrm{r}_{0}\right) \\
\tau_{0}\end{array}$ \\
\hline $\begin{array}{l}\text { Deformable mirror } \\
\text { fitting error }\end{array}$ & Higher DM spatial bandwidth & Low & $\begin{array}{l}\text { Residual seeing disk (outside OWA) } \\
\tau_{0} \\
\end{array}$ \\
\hline $\begin{array}{l}\text { Detector charge } \\
\text { diffusion }\end{array}$ & Improved detectors & Moderate & $\begin{array}{c}\text { N/A, depends on detector details } \\
\text { N/A }\end{array}$ \\
\hline $\begin{array}{l}\text { Direct scintillation } \\
\text { halo }\end{array}$ & $\begin{array}{l}\text { Two-conjugate phase or } \\
\text { amplitude correction }\end{array}$ & Simulations & $\begin{array}{l}\text { Scintillation scale }(\lambda / \sqrt{\lambda z}) \\
\text { High alt wind speed } / \sqrt{\lambda z}\end{array}$ \\
\hline $\begin{array}{l}\text { Dispersion } \\
\text { displacement }\end{array}$ & \begin{tabular}{|l|} 
Site selection \\
Lateral dispersion corrector \\
Optimized spectral width of \\
wavefront sensor \\
\end{tabular} & $\begin{array}{l}\text { Good } \\
\text { Concept } \\
\text { Concept }\end{array}$ & $\begin{array}{c}\text { Seeing disk }\left(\lambda / \mathbf{r}_{0}\right) \\
\tau_{0}\end{array}$ \\
\hline Flat-field instability & $\begin{array}{l}\text { Improved detectors and } \\
\text { calibration techniques }\end{array}$ & Moderate & $\begin{array}{c}\text { Depends on spatiotemporal power } \\
\text { spectrum }\end{array}$ \\
\hline Frozen wind lag & $\begin{array}{l}\text { Predictive phase correction of } \\
\text { multilayer atmosphere }\end{array}$ & Concepts & $\begin{array}{c}\text { Seeing disk }\left(\lambda / \mathbf{r}_{0}\right) \\
\tau_{0} \\
\end{array}$ \\
\hline $\begin{array}{l}\text { Higher-order terms in } \\
\text { the wavefront } \\
\text { expansion }\end{array}$ & $\begin{array}{l}\text { Better phase and amplitude } \\
\text { control, even outside the } \\
\text { habitable zone }\end{array}$ & Unknown & $\begin{array}{c}\text { N/A, couples light in from higher } \\
\text { spatial frequencies } \\
\text { Unknown decorrelation time }\end{array}$ \\
\hline Multispectral error & $\begin{array}{l}\text { Site selection } \\
\text { Common-band wavefront } \\
\text { sensing }\end{array}$ & $\begin{array}{l}\text { Good } \\
\text { Moderate }\end{array}$ & $\begin{array}{c}\text { Seeing disk }\left(\lambda / \mathrm{r}_{0}\right) \\
\tau_{0}\end{array}$ \\
\hline $\begin{array}{l}\text { Non-common path } \\
\text { calibration errors }\end{array}$ & $\begin{array}{l}\text { Common-path WFS/science } \\
\text { Calibration techniques }\end{array}$ & $\begin{array}{l}\text { Concept } \\
\text { Poor }\end{array}$ & $\begin{array}{l}\text { Depends on spatiotemporal power } \\
\text { spectrum }\end{array}$ \\
\hline Polarization effects & $\begin{array}{l}\text { Slow optics, two polarization } \\
\text { correction }\end{array}$ & Unknown & $\begin{array}{c}\text { Unknown halo width } \\
\text { Depends on observing technique }\end{array}$ \\
\hline Scintillation in WFS & $\begin{array}{l}\text { Amplitude correction } \\
\text { Robust WFS design }\end{array}$ & $\begin{array}{l}\text { Simulations } \\
\text { Concept }\end{array}$ & $\begin{array}{l}\text { Outer working angle }(\lambda / \mathrm{dx}) \\
\text { High alt wind speed } / \sqrt{\lambda z}\end{array}$ \\
\hline $\begin{array}{l}\text { Uncorrectable } \\
\text { telescope errors }\end{array}$ & $\begin{array}{l}\text { Improved telescope stiffness } \\
\text { and active control }\end{array}$ & Moderate & $\begin{array}{c}\text { Depends on spatiotemporal power } \\
\text { spectrum }\end{array}$ \\
\hline WFS aliasing & $\begin{array}{l}\text { Spatial filtering for pupil-plane } \\
\text { WFS } \\
\text { Focal-plane WFS }\end{array}$ & $\begin{array}{l}\text { Simulations } \\
\text { Concepts } \\
\end{array}$ & $\begin{array}{c}\mathrm{N} / \mathrm{A} \text {, causes leakage into OWA } \\
\tau_{0}\end{array}$ \\
\hline $\begin{array}{l}\text { WFS calibration } \\
\text { instability }\end{array}$ & $\begin{array}{l}\text { Wavefront sensors not sensitive } \\
\text { to changes in seeing } \\
\text { Active thermal control }\end{array}$ & $\begin{array}{l}\text { Moderate } \\
\text { Moderate }\end{array}$ & $\begin{array}{l}\text { Depends on spatiotemporal power } \\
\text { spectrum }\end{array}$ \\
\hline $\begin{array}{l}\text { WFS guide star and } \\
\text { sky photon noise }\end{array}$ & $\begin{array}{l}\text { Sensor design optimized for } \\
\text { habitable zone clearing }\end{array}$ & Concepts & $\begin{array}{c}\text { Outer working angle }(\lambda / \mathrm{dx}) \\
\text { WFS sample time dt }\end{array}$ \\
\hline $\begin{array}{l}\text { WFS read and dark } \\
\text { current noise }\end{array}$ & Improved detectors & Moderate & $\begin{array}{c}\text { Outer working angle }(\lambda / \mathrm{dx}) \\
\text { WFS sample time } d t\end{array}$ \\
\hline
\end{tabular}

Table 1. Physical error processes that can result in residual atmospheric wavefront phase and amplitude fluctuations in the science path.. For a more detailed discussion of some of these error terms, see Dekany ${ }^{16}$, et al., 2004. 
In this equation a and $\phi$ represent the transform of the aperture and phase functions $A$ and $\Phi$, respectively. The first term corresponds to theoretical diffraction from the aperture; the third and fourth terms are also proportional to the amplitude spread function and are similarly suppressed with diffraction. The second term, however, is of most interest and corresponds to the power spectrum of residual wavefront errors.

Expanding the wavefront errors as the sum of independent error processes, and assuming the long-exposure PSF is the sum of long-exposure PSFs due to each error term, we can write

$$
p_{\text {total }}=a \otimes\left(\sum_{i}\left|\Phi_{i}\right|^{2}+\left|A^{\prime}\right|^{2}\right)
$$

where the $\phi_{i}$ represent the phase spectra and $A_{i}$ the amplitude spectra of each error term, as appropriate.

The detailed shape of the various residual error power spectra will lead to detection thresholds that are, in general, a function of location in the focal plane. For simplicity, however, we will here assume that all residual power spectra are white within the outer working angle. In this model, an exoearth can be detected with the same signal-to-noise ratio (SNR) anywhere in the cleared-out portion of the halo. Detailed calculation that overcome this simplification are required to more accurately determine the practical science reach for high-contrast with TMT.

\subsubsection{Residual wavefront power spectra}

The strength of these white power spectra will depend both on the intrinsic phase energy and the area of the focal plane over which the corresponding halo of speckles is distributed. The mean-squared phase variance for each term can be obtained from scaling laws that can be found in a number of references on astronomical adaptive optics, most notably the text by Hardy ${ }^{17}$. The general form of each power spectrum is therefore,

$$
\left|\phi_{i}\right|^{2}=\left(\sigma_{i}^{2} /\left(4 w_{i}^{2}\right)\right) \Pi\left(f_{x} w_{i}\right) \Pi\left(f_{y} w_{i}\right)
$$

where $\sigma^{2}{ }_{i}$ is the mean-squared phase error of the $i^{\text {th }}$ process, $w_{i}{ }^{2}$ is the halo width (diameter), $\Pi$ is the rectangle function of Bracewell ${ }^{18}$, and $\left(f_{x}, f_{y}\right)$ is spatial frequency.

\section{SIGNAL-TO-NOISE RATIO AND INTEGRATION TIME}

Building upon the developments by Racine et al. ${ }^{19}$ and Macintosh et al. ${ }^{20}$, we can write the signal-to-noise ratio (SNR) for high contrast detection as,

$$
S N R=\frac{\text { Planet signal }}{\sqrt{\sigma_{\text {sky photon }}^{2}+\sigma_{\text {planet photon }}^{2}+\sigma_{\text {halo photon }}^{2}+\sigma_{\text {halo speckles }}^{2}}}
$$

where we differentiate between variances due to photon noise (the first three terms in the denominator) and speckle noise, the impact of the fact that most wavefront error processes lead to highly structured speckle structure in the PSF halo. It can be shown that this can be rewritten as, 


$$
S N R^{2}=\frac{\left(S F_{p} A\right)^{2}}{F_{s k y} A+S F_{p} A+\left(F_{s}^{s c i}\right) \sum_{i}^{\text {error process }}\left(\frac{\sigma_{i}^{2}}{N_{i}^{\text {speckles }}}\right)+\frac{\left(F_{s}^{s c i}\right)^{2}}{16} \sum_{i}^{\text {error process }}\left(\frac{\sigma_{i}^{4} \tau_{i}}{N_{i}^{\text {speckles }}}\right)} T
$$

where $\mathrm{S}$ is the Strehl ratio of correction, $\mathrm{F}_{\mathrm{p}}$ is the planet flux, $\mathrm{A}$ is collecting area of the entire telescope, $\mathrm{F}_{\text {sky }}$ is the sky background flux in a resolution element, $\mathrm{F}_{\mathrm{s}}^{\text {sci }}$ is the flux from the star as measured in the science band, $\sigma^{2}{ }_{i}$ is the wavefront variance used above, $\mathrm{N}_{i}^{\text {speckles }}$ is the number of speckles in the halo of process $\mathrm{i}, \tau_{\mathrm{i}}$ is the decorrelation time of halo speckles, and $\mathrm{T}$ is the total integration time.

Solving the above equation for integration time, we find,

$$
T \approx T_{\text {planet }}+\sum_{i}^{\text {error process }} T_{\sigma_{i}}^{\text {photon }}+\sum_{i}^{\text {error process }} T_{\sigma_{i}}^{\text {speckle }}=\sum_{i}^{\text {error process }} T_{\sigma_{i}}
$$

which shows that the total integration time required to reach a given SNR is the additive sum of times needed to reach that SNR in the presence of each of the error processes individually.

\section{Q VALUE}

The high-contrast image quality parameter, $\mathrm{Q}$, is defined at the ratio between the unwanted stellar halo flux and the desired planet flux, within a single resolution element. For $\mathrm{Q}>=1$, postprocessing is straightforward as the planet signal can be easily seen above the stellar halo. Unfortunately, residual atmospheric wavefront errors preclude suppression of the stellar halo to this level. Ground-based telescopes typically must work at small to very small $Q$ values. This places challenging requirements on calibration of the residual halo, so that the small planet signal (which may have reasonable SNR) can be 'pulled out' of the brighter halo. Although theoretically observations at an arbitrarily small Q value could be calibrated, in practice no $\mathrm{AO}$ system to date has reported ${ }^{21}$ detections below $\mathrm{Q}=0.5$ although some analysis has shown that an order of magnitude better performance might be possible with well-calibrated ground based adaptive optics systems ${ }^{22}$.

$Q$ can be calculated ${ }^{16}$ for each wavefront error process having mean-square phase variance $\sigma_{i}^{2}\left[\operatorname{rad}^{2}\right]$ and producing a halo of width, $\mathrm{w}_{\mathrm{i}}$, in the focal plane. For a telescope with diameter $\mathrm{D}$ and for imaging contrast $\mathrm{C}_{\text {planet, }}$

$$
Q=I_{p} / I_{\text {halo }}=\sigma_{\text {phase }}{ }^{-2}\left(\Omega_{\text {halo }} / \Omega_{\text {tel }}\right) C_{\text {planet }}=\sigma_{i}^{-2}(D / w)^{2} C_{\text {planet }}
$$

Typical values of $\mathrm{Q}$ for optimized high-contrast $\mathrm{AO}$ systems with $\mathrm{D}=30 \mathrm{~m}$ and $\mathrm{C}_{\text {planet }}=1.7 \times 10^{-10}$ are on the order of 0.0005 . In other words, the small planet signal must be pulled out of an evolving stellar halo that, on average, has two thousand times as large surface brightness. Successful calibration, therefore, requires excellent control of systematic errors, but does not theoretically preclude exoearth study.

\section{SCIENCE REACH FOR TMT}

Using this parametric model, we can now calculate the integration time required to study an exoearth analogue at 10pc, making a number of assumptions:

- A clear, high mountain site, with ambient pressure, $P_{0}=0.6$ atmospheres.

- Wavefront sensing and science both performed in either R-band, center $0.7 \mu \mathrm{m}$, bandwidth of $39 \%(0.27 \mu \mathrm{m})$ or $\mathrm{H}$-band, center $1.65 \mu \mathrm{m}$, bandwidth of $24 \%(0.40 \mu \mathrm{m})$, as two distinct cases to be considered

- $\quad$ WFS transmission $=0.1$ and science path transmission $=0.1$ 
- A Kolmogorov atmosphere with $\mathrm{r}_{0}=25 \mathrm{~cm}$ at $0.5 \mu \mathrm{m}$ wavelength at 45 degree zenith angle, $\zeta$. This corresponds to $\mathrm{r}_{0}=37.5 \mathrm{~cm}$ at R-band and $105 \mathrm{~cm}$ at $\mathrm{H}$-band.

- An atmospheric correlation time, $\tau_{0}=7.7 \mathrm{~ms}$ at R-band and $21.8 \mathrm{~ms}$ at H-band.

and the following assumptions relevant to the individual high-contrast error terms:

\begin{tabular}{|c|c|}
\hline Effect & Parameter / Assumption \\
\hline \multirow[t]{2}{*}{ Boiling wind } & $\sigma_{\text {boil lag }}^{2}=0.5 *\left(3.2 \mathrm{dt} / \tau_{\text {boil }}\right)^{5 / 3}\left[\mathrm{rad}^{2}\right]$ \\
\hline & $\tau_{\text {boil,R-band }}=71 \mathrm{~ms} ; \tau_{\text {boil, H-band }}=200 \mathrm{~ms}$ \\
\hline Chromatism & Common-band wavefront sensing and science \\
\hline Deformable mirror fitting error & None / Assumed perfectly eliminated within outer working angle (OWA) \\
\hline Detector charge diffusion & No charge diffusion \\
\hline \multirow[t]{2}{*}{ Direct scintillation halo } & $\begin{aligned} \sigma_{\text {scint, R-band }}{ }^{2}= & 0.0289 ; \sigma_{\text {scint, } \mathrm{H} \text {-band }}{ }^{2}=0.0106 \text { at } \zeta=45 \mathrm{deg} \\
& \text { Propagation length, } \mathrm{z}=8.3 \mathrm{~km}\end{aligned}$ \\
\hline & $\tau_{\text {scint }, \mathrm{R} \text {-band }}=16 \mathrm{~ms} ; \tau_{\text {scint } \mathrm{H} \text {-band }}=24 \mathrm{~ms}$ \\
\hline Dispersion displacement & $\begin{array}{c}1 / 2 \sec ^{8 / 3}(\zeta) \tan ^{5 / 3}(\zeta)\left(\mathrm{P}_{0} /\left(\mathrm{g} \rho_{\mathrm{s}}\right)\right)^{5 / 3} * \mathrm{G}^{*} \mathrm{H} \\
\tau_{0, \text { R-band }}=7.8 \mathrm{~ms}, \tau_{0, \text { H-band }}=21.8 \mathrm{~ms}\end{array}$ \\
\hline Flat-field instability & Perfectly stable flat-fields \\
\hline Frozen wind lag & Perfect phase prediction \\
\hline $\begin{array}{l}\text { Higher-order terms in the wavefront } \\
\text { expansion }\end{array}$ & $\begin{array}{c}\text { All higher-order terms negligible } \\
\text { (in practice we restrict total wavefront Strehl }>0.96 \text { ) }\end{array}$ \\
\hline Multispectral error & Common-band wavefront sensing and science \\
\hline Non-common path calibration errors & Perfect non-common path calibration \\
\hline Planet photon noise & Contrast of $1.7 \times 10^{-10}$ \\
\hline Polarization effects & No polarization effects \\
\hline Scintillation in WFS & Perfect amplitude correction \\
\hline Sky photon noise & Sky brightness $m_{R}=21.5 \operatorname{arcsec}^{-2} ; m_{H}=16 \operatorname{arcsec}^{-2}$ \\
\hline Uncorrectable telescope errors & No uncorrectable telescope errors \\
\hline WFS aliasing & Perfect filtering or focal-plane wavefront sensing \\
\hline WFS calibration instability & Perfectly stable WFS calibration \\
\hline WFS guide star and sky photon noise & $\begin{array}{c}\text { Guide star magnitude } \mathrm{m}_{\mathrm{R}}=4.2 ; \mathrm{m}_{\mathrm{H}}=3.2 \\
\text { Decorrelation time for WFS noise speckles }=\mathrm{dt}\end{array}$ \\
\hline WFS read and dark current noise & 4 pixels $/$ measurement, $\sigma_{\text {read, R-band }}=3 \mathrm{e}-\mathrm{rms}, \sigma_{\text {read, H-band }}=15 \mathrm{e}-\mathrm{rms}$ \\
\hline
\end{tabular}

Table 2. Model assumptions to determine the fundamental limits to exoearth study with TMT. Here, $g$ is acceleration due to gravity $=9.8 \mathrm{~m} \mathrm{~s}^{-2}, \rho_{\mathrm{s}}$ is the density of air at standard temperature and pressure, $\mathrm{G}$ is a weighting function that depends on the color of the guide star, here assumed to enjoy lateral dispersion correction ${ }^{23}$ We assumed $\mathrm{G}_{\mathrm{R} \text {-band }}=6.5 \times 10^{-11}$ and $\mathrm{G}_{\mathrm{H} \text {-band }}=6.3 \times 10^{-12}$. $\mathrm{H}$ is a weighting function that depends on the details of the $\mathrm{C}_{\mathrm{n}}{ }^{2}$ distribution with height,. We assume $\mathrm{H}_{\mathrm{R} \text {-band }}=5.7$ radians $^{2} \mathrm{~m}^{-5 / 3}$ and $\mathrm{H}_{\mathrm{H} \text {-band }}=1.0$ radians $^{2} \mathrm{~m}^{-5 / 3}$.

Furthermore, we will allow the WFS subaperture dimension, $\mathrm{dx}$, and temporal sample period, dt, to be free parameters to be optimized during the design process. All error sources affecting the exoplanet Strehl ratio, particularly fitting error, will be included in the optimization of SNR. This tends to drive the optimum deformable mirror spatial bandwidth higher than would be found using only the stellar halo assumptions in Table 2.

The actual deformable mirror spatial bandwidth will also be influenced by the assumption that the overall Strehl performance is sufficiently high to justify the second-order phase expansion above. Furthermore, although the Nyquist criterion states that spatial frequencies up to $\mathrm{N} / 2$ cycles per aperture, where $\mathrm{N}$ is the number of deformable mirror elements across the pupil, are theoretically correctable, it is not in general possible to correct two orthogonal phasors (e.g. simultaneously correct a sine wave and cosine wave both possessing the critical spatial frequency). This will also tend to drive up the required spatial bandwidth of the deformable mirror. Even so, we will find that deformable mirrors 
having actuator pitch of $\sim 10 \mathrm{~cm}$ or more projected on the entrance pupil are sufficient for this application. Thus total actuator counts of a few times $10^{4}$ are reasonable goals for high-contrast system development. There is relatively weak dependence on the choice of subaperture diameter, dx, as the WFS noise error terms that depend most critically upon WFS photon collecting area, $\mathrm{dx}^{2}$, create residual speckle haloes whose areas vary inversely with WFS collecting area, as $\mathrm{dx}^{-2}$.

The results of this analysis are presented in Table 3 .

\begin{tabular}{|c|c|c|}
\hline \multirow[t]{2}{*}{ Effect } & \multicolumn{2}{|c|}{ Integration time [hours] } \\
\hline & R-band & H-band \\
\hline Planet photon noise & 0.001 & 0.0002 \\
\hline Sky photon noise & 0.00003 & 0.0013 \\
\hline WFS photon noise & 2.1 & 0.12 \\
\hline Dispersion displacement & 0.2 & 0.008 \\
\hline Boiling wind & 0.5 & 0.04 \\
\hline Fundamental Error Total & $\begin{array}{c}2.8 \\
(\mathrm{dx}, \mathrm{dt})_{\mathrm{opt}}=(10 \mathrm{~cm}, 0.08 \mathrm{~ms})\end{array}$ & $\begin{array}{c}0.2 \\
(\mathrm{dx}, \mathrm{dt})_{\mathrm{opt}}=(12 \mathrm{~cm}, 0.04 \mathrm{~ms})\end{array}$ \\
\hline $\begin{array}{l}\text { Cumulatively adding WFS detector } \\
\text { read noise }\end{array}$ & $\begin{aligned} & 4.4 \\
&(\mathrm{dx}, \mathrm{dt})_{\mathrm{opt}}=(26 \mathrm{~cm}, 0.10 \mathrm{~ms})\end{aligned}$ & $\begin{aligned} & 0.4 \\
\left(\mathrm{dx}^{1}, \mathrm{dt}_{\mathrm{opt}}\right)= & (32 \mathrm{~cm}, 0.05 \mathrm{~ms})\end{aligned}$ \\
\hline $\begin{array}{l}\text { Cumulatively adding effect of } \\
\text { frozen wind lag error (no predictive } \\
\text { control) }\end{array}$ & $\begin{array}{c}6.8 \\
(\mathrm{dx}, \mathrm{dt})_{\mathrm{opt}}=(27 \mathrm{~cm}, 0.07 \mathrm{~ms})\end{array}$ & $\left(\mathrm{dx}^{1}, \mathrm{dt}_{\mathrm{opt}}\right)=\left(\begin{array}{l}2.3 \\
(32 \mathrm{~cm}, 0.03 \mathrm{~ms})\end{array}\right.$ \\
\hline $\begin{array}{l}\text { Cumulatively adding effect of } \\
\text { scintillation }\end{array}$ & $\begin{array}{c}35 \\
(\mathrm{dx}, \mathrm{dt})_{\mathrm{opt}}=(18 \mathrm{~cm}, 0.08 \mathrm{~ms})\end{array}$ & $\begin{array}{c}28 \\
(\mathrm{dx}, \mathrm{dt})_{\mathrm{opt}}=(32 \mathrm{~cm}, 0.04 \mathrm{~ms})\end{array}$ \\
\hline
\end{tabular}

Table 3. Integration time required for a $\mathrm{D}=30 \mathrm{~m}$ telescope imaging a contrast $1.7 \times 10^{-10}$ exoearth analogue in a $1 \mathrm{AU}$ orbit at $10 \mathrm{pc}$ at spectroscopic resolution $\mathrm{R} \sim 5$ to achieve an $\mathrm{SNR}=5$, under the assumptions presented above. The fundamental error total integration time considers the first five error terms. Subsequent integration times consider the impact of increasingly objectionable effects.

The dependence of integration time on the brightness of the guide star is shown, approximately, in Figure 1.

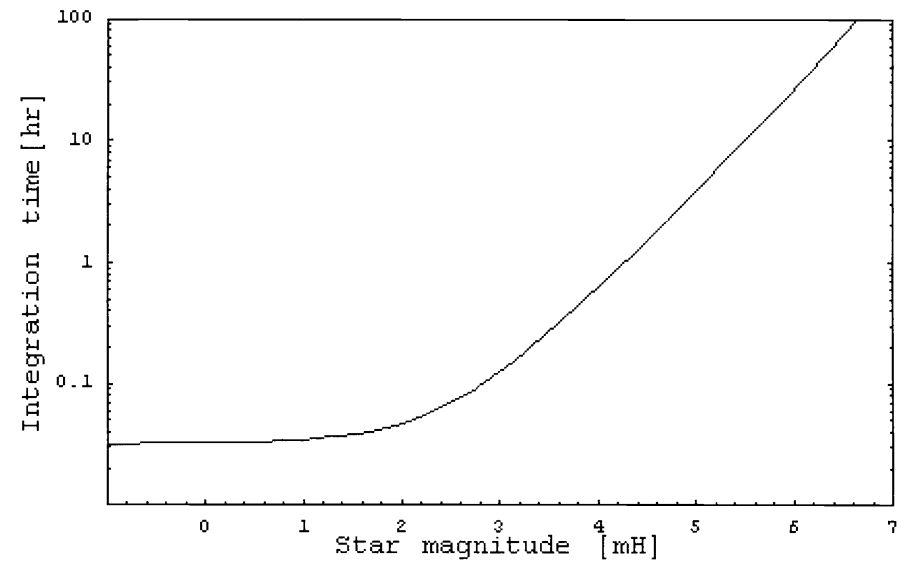

\footnotetext{
${ }^{1}$ Although optimization of the SNR equation in this case leads a larger value for $\mathrm{dx}_{\mathrm{opt}}$, we limit the size of $\mathrm{dx}$ here in order to satisfy the validity of the second-order phase expansion, which is violated for too large values of deformable mirror fitting error.
} 


\section{DISCUSSION}

The fundamental limitations imposed by photon noise, unpredictable boiling wind induced phase errors, and finite observation bandwidths allow the $\mathrm{SNR}=5$ study of exoearth analogues at $10 \mathrm{pc}$ in one long night of integration. This, however, requires the successful implementation of many new technologies and observing techniques as summarized in Table 1 and prioritized in Table 3.

This analysis provides a strategy for the mitigation of the most objectionable effects in a well-balanced AO system optimized for high-contrast. We find that the impact of scintillation (under current assumptions) is significant for exoearth study in both $\mathrm{R}$ band and $\mathrm{H}$ band and must therefore be actively compensated. Fortunately, because the impact on integration time depends on the log-amplitude variance (photon noise) and log-amplitude variance squared (speckle noise), even partial compensation of amplitude errors can dramatically reduce required integration time. In this case, reduction of log-amplitude variance by $90 \%$ would be sufficient to make the scintillation contribution small compared to the fundamental errors.

Similarly, predictive wavefront control is another key technology that must be developed to reach the fundamental limits in both $\mathrm{R}$ and $\mathrm{H}$ bands. Again, even partial predictive correction would be very beneficial. The practical limitations on the quality of wavefront phase prediction in a multilayer, multivelocity atmosphere requires further research.

WFS read noise, particularly in $\mathrm{H}$ band, tends to drive wavefront sensing subapertures up in area, which threatens other assumptions in the current model (e.g. perfect antialiasing, negligible fitting error and higher-order wavefront error expansion terms). The constraints imposed by these assumptions, when evaluated in detail, are likely to drive up the impact of WFS read noise in fully optimized system designs.

\section{CONCLUSIONS}

\subsubsection{TMT exoearth target population}

From Figure 1 we see that the potential science reach, as described the fundamental error terms can be extended to stars of approximate apparent magnitude $\mathrm{m}_{\mathrm{H}}=5$. Thus, there are potentially scores of systems can could be searched for exoearths. Basic compositional questions regarding the presence or absence of an atmosphere or of unusual (nonterrestrial) spectral lines (e.g. steam lines) could be answered. In addition, potentially hundreds of lower contrast exojupiters and giant terrestrial planets (e.g. water worlds) are within the theoretical science reach of TMT.

For stars brighter than magnitude $\sim 3, \mathrm{SNR}=5$ integration times at $\mathrm{R} \sim 5$ are as small as a few minutes. For these systems $\mathrm{R} \sim 25$ study is possible, which could allow study of deep methane and water features in exoplanet atmospheres, particularly if TMT is constructed at a very dry site.

\subsubsection{Scientific return and limitation imposed by Earth's atmosphere}

A fundamental contradiction for ground-based studies of exoearth is that the more Earth-like their potential atmospheres and those atmosphere's chemistry becomes, the harder it is to study with ground based telescopes. Although techniques have been developed to study, for example, intergalactic water lines from Earth's surface, these techniques typically either rely on the Doppler shifting of narrow spectral lines as the Earth swings in its orbit around our Sun, or rely on differences in the temperature, pressure, or other physical environmental characteristic. Thus, temperature broadened steam lines can be studied from Earth, despite significant water content in our atmosphere.

Still, from the ground we can determine the presence of exoplanet atmosphere from the photometric return as a function of wavelength (from observed Rayleigh scatter), the potential existence of clouds or soot (from observed Mie scattering), evidence of rotational period and tidal locking (from repeated photometry), orbital resonances among sibling planets, equilibrium temperatures from their determined orbits, and strong $\mathrm{CH}_{4}$ lines. 
The key markers for extrasolar life, such as $\mathrm{O}_{2}$ and $\mathrm{O}_{3}$, are in general not available at the spectral resolutions and SNR's available with a 30 -meter ground based telescope.

\subsubsection{Implications for TMT research and development}

The results here demonstrate the theoretical capability of TMT to discover and study terrestrial planets in a significant number of nearby star systems. In order to achieve this potential, however, we must employ a class of adaptive optics systems unlike any previously developed. Because of this naïveté, the true challenge of mitigating the major performance drivers for high-contrast is not yet known. It is likely, however, that control of all of the error sources in Table 1 with sufficient quality to reach the fundamental performance limits will require a major research and development effort. A coordinated program to quantify and mitigate each of the potential error sources, with validation in the laboratory and in prototype systems on large telescopes is essential.

During conceptual design, it will be important to consider the impact of telescope design decisions in light of the impact upon the ultimate exoplanetary science obtainable with the TMT. Parametric models such as the one presented here, coupled with diffractive wave optics simulations capturing the appropriate physics, are needed immediately to allow scientific and engineering design optimizations to proceed.

\section{ACKNOWLEDGEMENTS}

This work has been supported by the Gordon and Betty Moore Foundation in support of the California Extremely Large Telescope (CELT) point design of the TMT. The author wishes to express thanks to Karl Stapelfeldt, Bruce Macintosh, and Wes Traub for many helpful discussions.

\section{REFERENCES}

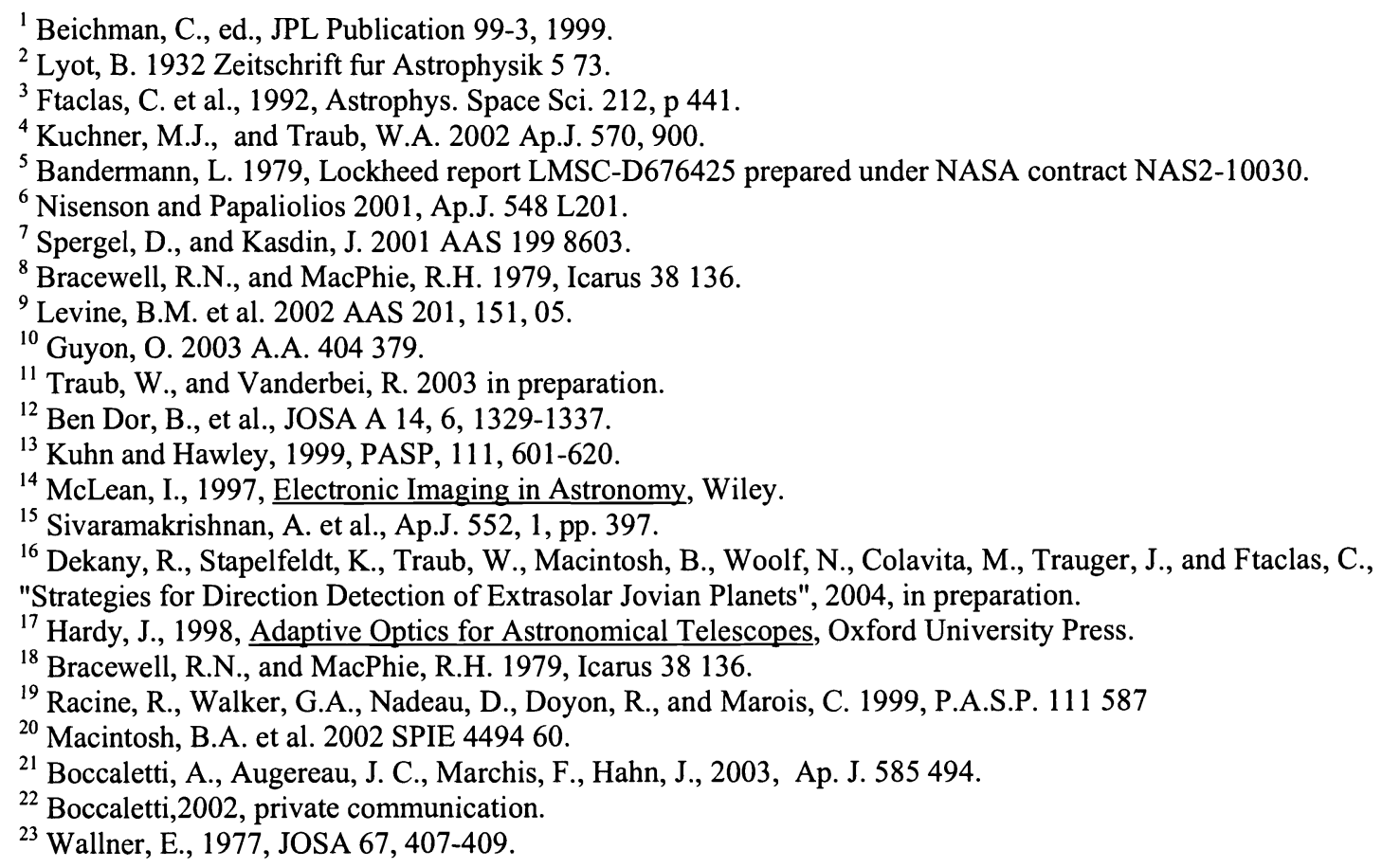

